\title{
A comparative study on the adverse effects of two anti- tuberculosis drugs regimen in initial two-month treatment period
}

\author{
Begum Lutfun Nahar'1, A. K. M. Mosharrof Hossain'1, M. Monirul Islam² and \\ Dipti Rani Saha ${ }^{1}$
}

${ }^{1}$ Department of Pharmacology and Therapeutics, Sylhet MAG Osmani Medical College, Sylhet 3100, Bangladesh; ${ }^{2}$ Chest Disease Hospital, Sylhet 3100, Bangladesh.

\begin{tabular}{l} 
Article Info \\
\hline Received: $\quad$ 4 July 2006 \\
Accepted: $\quad$ 13 September 2006 \\
Available Online: $\quad$ January 2008 \\
DOI: 10.3329/bjp.v1i2.488 \\
\\
\\
\\
Cite this article: \\
Nahar BL, Hossain AKMM, Islam \\
MM, Saha DR. A comparative study \\
on the adverse effects of two anti- \\
tuberculosis drugs regimen in initial \\
two-month treatment period. Bangla- \\
desh J Pharmacol. 2006; $1: 51-57$.
\end{tabular}

\section{Abstract}

Tuberculosis (TB) is a leading cause of death throughout the world and Bangladesh stands $4^{\text {th }}$ among high burden countries. Treatment of TB hampered with poor patient compliance and intolerance at least partly due to the adverse drug reactions. A prospective longitudinal non-randomized case study was conducted on 64 Hospital admitted patients diagnosed as primary (category I) and resistant or treatment failure (category II) to compare adverse effects between two anti-TB drug treatment regimen based on diagnostic category. Category I received four drug (rifampicin, isoniazid, ethambutol, pyrazinamide) and category II received five drug (rifampicin, isoniazid, ethambutol, pyrazinamide, sparfloxacin) combination treatments for initial 2 months under DOTS during the period of July 2004 to July 2005. Adverse effect parameters e.g. gastrointestinal disturbances, arthralgia, hepatic dysfunction and renal impairment were estimated before, two and eight weeks after initiation of treatment. Predisposing risk factors for adverse effects e.g. age, sex, nutritional status, associated disease, habits were also analyzed. In our study, $76.5 \%$ of total patients experienced some sorts of adverse effects. In 4- and 5-drug regimen groups' adverse reaction were observed in 50 and $95 \%$ of patients respectively. Serum bilirubin, SGPT, creatinine did not change in neither of the treated group while alkaline phosphatase tended to decrease and uric acid to increase. No disease was established to be risk factor for drug intolerance.

\section{Introduction}

Tuberculosis (TB) causes three million death each year (Wright et al., 1998), that is declared to be 'global health emergency' by World Health Organization (WHO, 1996). Bangladesh is one of the top five high burden countries in the world where TB is the second killer infectious disease next to diarrhea and about 0.6 million people are estimated to suffer from TB (Benoor et al., 1998). Despite the availability of affective chemotherapy TB is still a major health problem in most countries
(Grange, 1990). The poor outcome was attributed to poor patient compliance, to primary multidrug resistance and to interruption partly due to adverse drug reaction (WHO, 1997). The most important step to ensure treatment is the patients' adhere to treatment are introduction of direct observation treatment- short course (DOTS) and use of fixed dose combination, as recommended by WHO (WHO, 1998; Blomberg et al., 2001).

Anti-TB chemotherapy exhibit greater level of efficacy 
Table I

Adverse effects observed in course of two anti-tuberculosis drug treatment regimen recorded during initial two month of treatment

\begin{tabular}{|c|c|c|}
\hline Symptoms & $\begin{array}{c}\text { 4-drug group } \\
n=14\end{array}$ & $\begin{array}{c}5 \text {-drug group } \\
n=20\end{array}$ \\
\hline Nausea & $1(7.1)$ & $1(5)$ \\
\hline Vomiting & $1(7.1)$ & $1(5)$ \\
\hline Anorexia & $1(7.1)$ & $2(10)$ \\
\hline Abdominal pain & $1(7.1)$ & $6(30)$ \\
\hline Joint pain & $5(35.7)$ & $6(30)$ \\
\hline Joint swelling & 0 & $3(15)$ \\
\hline Muscle weakness & $2(14.3)$ & $5(40)$ \\
\hline Visual disturbances & $4(28.6)$ & $5(40)$ \\
\hline Rash & 0 & $3(15)$ \\
\hline Vestibular disturbances & $1(7.1)$ & 0 \\
\hline Peripheral neuropathy & 00 & $1(5)$ \\
\hline
\end{tabular}

Values in parenthesis are expressed in percentage

with an acceptable degree of toxicity, however combination treatment may produce severe adverse events (Schaberg et al., 1996). Important adverse effects are hepatitis, rash, gastrointestinal upset, hyperuricemia, peripheral neuropathy, visual disturbances (Pande et al., 1996; Hussain et al., 2003; Bass et al., 1994; Scott, 1995; Solangi et al., 2004; Pereira et al., 2000; Tsai and Lee, 1997).

A few studies are done on adverse effects of anti-TB drugs related to specific regimen of drug and risk factors for those adverse effects. So, we aimed to study the adverse drug reaction and to analyze risk factors for intolerance and adverse effects of two anti-TB drug regimen in initial two month phase of treatment.

\section{Materials and Methods}

A prospective longitudinal non-randomized case study was carried out on diagnosed pulmonary TB patients admitted in the Sylhet Chest Disease Hospital and Sylhet Shaheed Shamsuddin Ahmed Hospital during the period of July 2004 to July 2005.

Inclusion criteria were pulmonary tuberculosis patients diagnosed as primary treatment failure (category I) or relapse case or resistance TB (category II). Cases of either sex with age above 14 years and patients who were under DOTS. While patients with history of hepatitis, renal or hepatic impairment; pregnant women and age below 14 years were excluded from the study.

In total 34 patients were included in this study, of which 14 had received category I, 4 anti-TB drugs that include rifampicin, isoniazid, ethambutol, pyrazinamide. Another 20 patients received category II: 5 anti-
TB drugs that included rifampicin, isoniazid, ethambutol, pyrazinamide and sparfloxacin. All the patients received the drugs under direct observation therapy short-course (DOTS)-treatment program.

Primary variables were organ -related adverse effects e.g. signs, symptoms and function tests. Secondary variables were predisposing risk factors e.g. age, sex, nutritional status, socio-economic condition and habits.

Organ-related adverse effects and biochemical parameters were estimated before ( 0 week) and 4 and 8 weeks after initiation of treatment.

Data was analyzed by $x^{2}$, One-way ANOVA with repeated measure design and post hoc test of significance was done by pair t-test by using SPSS 11 .

\section{Results}

A total number of 64 patients were included of which 34 completed the study. Among the 30 patients who did not complete the study, 4 patients expired and 26 patients dropped out. Among the patients completed the study, $27(79.4 \%)$ were male and 7 (20.6\%) were female. In 4-drug treatment group 14 male and female patients of age $38.0 \pm 9.6$ years (mean \pm SD) and in 5drug treatment group 20 male and female patients of age $38.6 \pm 12.8$ years were included. The mean age of male and female patients was $38.4 \pm 11.6$ years and 38.1 \pm 13.9 years respectively. Male and female patients did not differ in their age $(\mathrm{t}=0.04, \mathrm{NS})$.

All of $27(100 \%)$ male patients and out of 7 female patients $5(71.4 \%)$, yielding a total number of $32(94.1 \%)$ were in under nutritional group and lived in low socio- 
Table II

Biochemical organ function tests of 4-drug treatment regimen group recorded during initial two month of

\begin{tabular}{|lrrr|}
\hline Biochemical tests & \multicolumn{1}{c}{ treatment } & \multicolumn{1}{c}{ week } & \multicolumn{1}{c|}{8 weeks } \\
\hline Serum bilirubin & $0.5 \pm 0.2$ & $0.6 \pm 0.8$ & $0.4 \pm 0.2$ \\
Serum ALT (SGPT) & $4.8 \pm 2.2$ & $7.5 \pm 6.0$ & $5.9 \pm 3.1$ \\
Serum alkaline phosphatase & $102.8 \pm 51.2$ & $78.5 \pm 24.1$ & $70.7 \pm 41.6$ \\
Serum creatinine & $1.1 \pm 0.4$ & $1.1 \pm 0.4$ & $1.0 \pm 0.2$ \\
Serum uric acid & $4.9 \pm 2.1$ & $6.2 \pm 2.7$ & $6.3 \pm 1.5$ \\
Serum glucose & $93.6 \pm 20.9$ & $99.2 \pm 20.7$ & $85.2 \pm 14.0$ \\
\hline
\end{tabular}

Table III

Biochemical organ function tests of 5-drug treatment group recorded during initial two month of treatment

\begin{tabular}{|lrrr|}
\hline Biochemical tests & \multicolumn{1}{c}{0 week } & \multicolumn{1}{c|}{4 weeks } & 8 weeks \\
\hline Serum bilirubin & $0.5 \pm 0.7$ & $0.7 \pm 1.3$ & $0.4 \pm 0.3$ \\
Serum ALT(SGPT) & $11.1 \pm 16.6$ & $11.5 \pm 8.9$ & $8.1 \pm 4.5$ \\
Serum alkaline phosphatase & $111.5 \pm 85.7$ & $106.3 \pm 55.9$ & $79.2 \pm 26.1$ \\
Serum creatinine & $0.9 \pm 0.3$ & $1.1 \pm 0.2$ & $1.1 \pm 0.2$ \\
Serum uric acid & $4.5 \pm 1.6$ & $5.6 \pm 2.1$ & $5.7 \pm 2.5$ \\
Serum glucose & $91.9 \pm 25.5$ & $86.0 \pm 27.2$ & $84.5 \pm 16.6$ \\
\hline
\end{tabular}

economic condition. In habitual status 10 (37.0\%) of males were smoker, while none of female had that habit. Eight $(29.6 \%)$ of male and 4 (57.1\%) female patients making a total number of $12(35.3 \%)$ were habituated with betel nuts.

Out of the treated patients $76.5 \%$ experienced some sorts of unwanted effects (Table I). Adverse effects were observed in 7 patients (50\%) in 4-drug regimen group and in 19 patients (95\%) in 5-drug regimen group. Notable adverse effects were abdominal pain experienced by 1 patient (7.1\%), muscle weakness by 2 patients $(14.3 \%)$ and joint pain by $5(35.7 \%)$ in 4 -drug regimen group. In 5-drug regimen group abdominal pain was experienced by $6(30 \%)$ patients, muscle weakness by $5(40 \%)$ and joint pain by $6(30 \%)$ patients respectively.

In the 4-drug treatment group before initiation of treatment regimen the estimated serum glucose level was $93.6 \pm 20.9 \mathrm{mg} / \mathrm{dL}$. After 2 and 8 weeks of inception of treatment regimen glucose level was detected as $99.2 \pm 20.7$ and $85.2 \pm 14.0 \mathrm{mg} / \mathrm{dL}$ respectively (Table II). These differences were not statistically significant.

In the 5-drug treatment group before initiation of treatment regimen the estimated serum glucose level was $91.9 \pm 25.5 \mathrm{mg} / \mathrm{dL}$. After 2 and 8 weeks of inception of treatment regimen creatinine level was detected as $86.0 \pm 27.2$ and $84.5 \pm 16.6 \mathrm{mg} / \mathrm{dL}$ respectively (Table III).

In the 4-drug treatment group before implementing the treatment regimen bilirubin was $0.5 \pm 0.2 \mathrm{mg} / \mathrm{dL}$. After 2 and 8 weeks of inception of treatment regimen bilirubin level was estimated $0.6 \pm 0.8$ and $0.4 \pm 0.2 \mathrm{mg} /$ $\mathrm{dL}$ respectively (Table II). One-way ANOVA shows no changes in course of treatment in this group $[\mathrm{F}(2,39)=$ 0.952, NS].

In the 5-drug treatment group before initiation of the treatment regimen bilirubin (mean \pm SD) was $0.6 \pm 0.7$ $\mathrm{mg} / \mathrm{dL}$. After 2 and 8 weeks of inception of treatment regimen bilirubin level was estimated $0.7 \pm 1.3$ and $0.4 \pm$ $0.3 \mathrm{mg} / \mathrm{dL}$ respectively (Table III). One-way ANOVA shows no changes in course of treatment in this group $[\mathrm{F}(2,57)=0.547, \mathrm{NS}]$.

In the 4-drug treatment group before administration of the treatment regimen SGPT was $4.8 \pm 2.2 \mathrm{U} / \mathrm{L}$. After 2 and 8 weeks of treatment regimen SGPT level was estimated $7.5 \pm 6.0 \mathrm{mg} / \mathrm{dL}$ and $5.9 \pm 3.1 \mathrm{U} / \mathrm{L}$ respectively (Table II). One-way ANOVA shows no changes in SGPT in this group $[\mathrm{F}(2,39)=1.46, \mathrm{NS}]$. After two weeks of treatment SGPT tended to increase but was neither statistically significant nor clinically appreciable $[\mathrm{t}=1.4 ; \mathrm{df} 13, \mathrm{NS}]$. After 8 weeks of treatment SGPT level was almost same as that of pre- 
treatment level [ $\mathrm{t}=1.1 ; \mathrm{df} 13 ; \mathrm{NS}$ ]. There observed no difference between SGPT level estimated 2 and 8 weeks of treatment $[\mathrm{t}=0.9$; $\mathrm{df} 13 ; \mathrm{NS}]$.

In the 5-drug treatment group before initiation of the treatment regimen SGPT was $11.1 \pm 16.6 \mathrm{U} / \mathrm{L}$. After 2 and 8 weeks of inception of treatment regimen SGPT level was estimated $11.5 \pm 8.9$ and $8.1 \pm 4.5 \mathrm{U} / \mathrm{L}$ respectively (Table III). One-way ANOVA shows no changes in course of treatment in this group $[\mathrm{F}(2,57)=$ 0.569, NS]. There observed no difference between SGPT level estimated 2 and 8 weeks of treatment $[\mathrm{t}=1.6$; $\mathrm{df}$ $19 ;$ NS]. No differences in SGPT level was observed in the group of patients (4-drug vs 5-drug) when estimated after 2 weeks $[\mathrm{t}=1.5 ; \mathrm{NS}]$ and 8 weeks $[\mathrm{t}=$ $1.5 ; \mathrm{NS}]$.

In the 4-drug treatment group before implementing treatment regimen serum alkaline phosphatase was $102.8 \pm 51.2 \mathrm{U} / \mathrm{L}$. After 2 and 8 weeks of inception of treatment regimen alkaline phosphatase tended to decrease to $78.5 \pm 24.1$ and $70.7 \pm 41.6 \mathrm{U} / \mathrm{L}$ respectively (Table II). However, One-way ANOVA shows no significant differences in alkaline phosphatase in course of treatment $[\mathrm{F}(2,39)=2.392, \mathrm{NS}]$.

In the 5-drug treatment group before administration of treatment regimen serum alkaline phosphatase was $115.5 \pm 85.7 \mathrm{U} / \mathrm{L}$. After 2 and 8 weeks of inception of treatment regimen alkaline phosphates was estimated to be as $106.3 \pm 55.9$ and $79.2 \pm 26.1 \mathrm{U} / \mathrm{L}$ respectively (Table III). However, One-way ANOVA shows no significant differences in alkaline phosphatase in course of treatment $[\mathrm{F}(2,57)=1.91$, NS]

In the 4-drug treatment group before initiation of treatment regimen the estimated creatinine level was $1.1 \pm 0.4 \mathrm{mg} / \mathrm{dL}$. After 2 and 8 weeks of inception of treatment regimen creatinine level was detected as $1.1 \pm$ 0.4 and $1.0 \pm 0.2 \mathrm{mg} / \mathrm{dL}$ respectively (Table II).

In the 5-drug treatment group before initiation of treatment regimen the estimated creatinine level was $1.0 \pm 0.3 \mathrm{mg} / \mathrm{dL}$. After 2 and 8 weeks of inception of treatment regimen creatinine level was detected as $1.1 \pm$ 0.2 and $1.1 \pm 0.2 \mathrm{mg} / \mathrm{dL}$ respectively (Table III).

In the 4-drug treatment group before implementing the treatment regimen serum uric acid level was $4.9 \pm 2.1$ $\mathrm{mg} / \mathrm{dL}$. After 2 and 8 weeks of inception of treatment regimen uric acid level was detected $6.2 \pm 2.7$ and $6.3 \pm$ $1.5 \mathrm{mg} / \mathrm{dL}$ (Table II). After 2 weeks of treatment uric acid tended to increase but was not statistically significant $[\mathrm{t}=2.1$; df 13 , NS]. After 2 months of treatment uric acid level significantly increased in this treatment group [ $\mathrm{t}=2.3$, df 13 ; NS]. There observed no difference between uric acid level estimated 2 and 8 weeks of treatment $[t=0.2$, df 13; NS].

In the 5-drug treatment group before administration of the treatment regimen serum uric acid level was $4.5 \pm$ $1.6 \mathrm{mg} / \mathrm{dL}$. After 2 and 8 weeks of inception of treatment regimen uric acid level was detected 5.6 \pm 2.1 and $5.7 \pm 2.5 \mathrm{mg} / \mathrm{dL}$ (Table III). After 2 weeks of treatment uric acid tended to increase but was not statistically significant $[\mathrm{t}=2.1 ; \mathrm{df} 19, \mathrm{NS}]$. After 2 months of treatment uric acid level did not change [ $t=1.9$, df 19; NS].

\section{Discussion}

In 1998 the global TB program of WHO established Global TB Research initiative to support related research. Research has been done by NGOs on Health System and Service Research that include studies on DOTS by community health workers (Chowdhury et al., 1997), on microscopic diagnosis (Vandeun and Portalels, 1998) and drug resistance surveillance (Haque et al., 1995).

Compliance with anti-TB medication is essential to effective management. Two strategies to ensure compliance are DOTS and fixed dose combination (FDC) (Van leuven et al., 1997). Our study fully applied on DOTS strategy. Generally these drugs are well tolerated (Dutt et al., 1983), may be associated with unwanted effects of different origin. Adverse drug reactions had been extensively studied and reviewed (Haque and Golam Nabi, 1999; Mohan et al., 2004; Resi et al., 2004; Maddrey, 2005).

The present prospective study was undertaken to demonstrate the extent of adverse effects of two antituberculosis drug treatment regimens under DOTS. Adverse effects were assessed according to sign and symptoms of the patients and the systemic organrelated adverse effects were estimated by serum glucose, serum bilirubin, serum alanine aminotransferase, alkaline phosphatase, creatinine, uric acid. As predisposing risk factors for adverse effects we observed age, sex, nutritional status, socioeconomic condition and habit. Serum glucose was estimated before the treatment initiation with the aim to assess hyperglycemia as predisposing risk factor for $\mathrm{TB}$ or adverse drug events involving hepatic or renal dysfunction. Subsequently in course of treatment serum glucose level was monitored with the view of effects of these drugs on carbohydrate metabolism of liver. In our observation in course of treatment there were no significant changes in glucose level estimated neither before nor after two month of treatment. Most of the TB patients complete treatment without any significant adverse effects, however data (Ormerod and Horsfield, 1996) showed that side effects occur in $57.8 \%$ patients, while we observed $76.5 \%$ of patients experienced some sort of unwanted effects. Unwanted effects were minor in nature, only $2.9 \%$ exhibited major adverse effects e.g. 
hepatitis in our study, while Shakya et al. (2004) reported an $8 \%$ incidence of hepatotoxicity. Side effects related to the digestive system were observed in $29.4 \%$ of patients that was consistent with the observation of others (Ormerod and Horsfield, 1996). We observed no evidence of allergic reactions, neurotoxicity, cardiovascular-related adverse effects as observed by other (Ormerod and Horsfield, 1996). It is important that patients should be monitored during treatment so that adverse effects can be detected promptly and managed properly (WHO, 2003). Serious adverse effects require documented change in therapy or hospitalization. If minor adverse effects develop patients should continue with reduced doses and receive symptomatic treatment, if major side effects develop the offending drug should be stopped (WHO, 2003). The withdrawal of treatment due to major adverse effect e.g. hepatitis in 1.8 to $6.0 \%$ patients (Girling, 1978; Smith and Zirk, 1961). Ormerod and Horsfield (1996) reported that $51 \%$ had reaction to antituberculosis drug requiring modification of treatment while none of our patients required modification of treatment. Termination of treatment either with INH, rifampicin or pyrazinamide because of severe side effects was necessary in $23 \%$ as reported by Schaberg et al. (1996).

Baseline studies of complete blood counts, hepatic and renal function studies were suggested to be performed at the initiation of treatment (Dutt et al., 1983). In our study hepatic and renal function tests were done with the view to assess the effects of the used drugs on these organs. We observed no substantial abnormality in baseline liver and renal function tests in course of treatment regimens.

It is very uncommon to have adverse reactions to a single anti-TB drug, but such reaction with more than one is very small (Smith and Zirk, 1961), while adverse reactions to multiple drugs were reported (Mathus et al., 1979; Thamari and Gupta, 1981). In our observation, major side effects as hepatitis developed in $2.9 \%$, that was similar to the report of Dutt et al., 1983).

During the initial phase of 4-drug combination treatment regimen side effects were reported in $22.4 \%$ of patients (Haque and Golam Nabi, 1999).

In our 4-drug treatment regimen study group, $50 \%$ of patients developed side effects during the initial phase. Of them $35.7 \%$ complained of arthralgia, $28.6 \%$ experienced GIT upset, muscle weakness evidenced in $14.3 \%$, vestibular disturbances in $7.1 \%$ persons and sign of peripheral neuropathy was absent. Our observation differed to some extent from observation of others (Dutt et al., 1983; Ohkawa et al., 2002).

In 5-drug treatment regimen study group, adverse effects observed in $90 \%$ of patients. Of them $30 \%$ developed arthalgia, 50\% experienced GIT upset, 40\% complained of muscle weakness. Adverse effects showed commoner in 5-drug treatment group compared to 4-drug group was not statistically significant. In our study $21.4 \%$ patients exhibited a transient and symptomless increase of SGPT that was in agreement with other reports (Lee, 1995; Lee et al., 2002). Fattinger et al. (1997) reported eight times increase of SGPT, while we observed 2-3 times increase in a few patients. Incidence of hepatitis was reported in $7.7 \%$ with combination of use of rifampicin and pyrazinamide (Lee et al., 2002), compared to drug used alone viz. with pyrazinamide $15 \%$, with isoniazid $7 \%$, with rifampicin $1.5 \%$ and hepatitis was suggested due to pyrazinamide rather than isoniazid or rifampicin (Schaberg et al., 1996). Isoniazid and pyrazinamide combination administration was associated with an increased mortality in patients with hepatitis. Hepatotoxic potential of rifampicin or pyrazinamide is far less in the doses used in the modern day shortcourse regimen. We did not observe such results in our study.

We analyzed serum uric acid and creatinine level to assess kidney functions. Increase of uric acid level was reported (Solangi et al., 2004; Zierski and Bek, 1980). In our study $55.9 \%$ of patients showed increased uric acid level, that confirms other studies.

Ethambutol is also reported to increase uric acid level (Postlethwaite et al., 1971).

We did not observe any change in serum creatinine level, while Solangi et al. (2004) observed nonsignificant elevation. Our observation suggests no appreciable effects of anti-TB drugs on kidneys.

Risk factors for anti-TB drugs-induced hepatitis had been studied (Shakya et al., 2004; Fauzi et al., 2004; Marti et al., 2005; Tost et al., 2005). Case control studies were undertaken to assess the risk factors for hepatotoxicity with variable like age, sex, BMI, acetylator status, extent of disease, protein, alcohol intake (Pande et al., 1996). In our study hepatotoxicity developed in younger males who were given PZA that also is observed by Ohkawa et al. (2002). As predisposing risk factors there is no suggestive finding in our observation related to age and habit, however we observed TB more common in male; in poor nutritious and low socio-economic status.

Our study applied on DOTS of both 4-drug and 5 drugregimen patients. Due to time constrains and limitation of facilities the obtained results may not reflect the actual effects of drugs on population. Further prospective longitudinal interventional randomized studies with larger sample size of different subject group are necessarily recommended. 


\section{References}

Bass JB, Farer LS, Hopewell PC, O'Brien R, Jacobs RF, Ruben F, Snider DEJ, Thornton G. Treatment of tuberculosis and tuberculosis infections in adults and children. Am J Respir Crit Care Med. 1994; 149: 1359-74.

Benoor KS, Mahmood AM, Huq AKMS. Multidrug resistant tuberculosis: Problem arise. Abstract, The National Conference of Chest; 1998, p 15.

Blomberg B, Spinaci S, Fourie B, Laing R. The rationale for recommending fixed-dose combination tablets for treatment of tuberculosis. WHO. 2001; 79: 61-68.

Chowdhury AM, Chowdhury S, Islam MN, Islam A, Vaughan JP. Control of tuberculosis by community health workers in Bangladesh. Lancet 1997; 350: 169-72.

Dutt AK, Moers D, Stead WW. Undesirable side-effect of isoniazid and rifampicin in largely twice-weekly shortcourse chemotherapy for tuberculosis. Am Rev Respir Dis. 1983; 128: 419-24.

Fattinger K, Braunschweig S, Reichen J, Meier-Abt PJ, Krahenbuhl S. Liver injury under tuberulostatic treatment. Schweiz Rundsch Med Prax. 1997; 86: 626-29.

Fauzi AR, Shah A, Rathor MY, Satwi S, Risk factors for antituberculous drugs-induced hepatitis: A prospective survey from a chest clinic in a general hospital. Med J Malaysia. 2004; 59: 72-77.

Girling DJ. The hepatictoxicity of antituberculosis regimens containing isoniazid, rifampicin and pyrazinamide. Tubercle 1978; 59: 13-32.

Grange JM. Drug resistance and tuberculosis elimination. Bull Int Union Tuberc Lung Dis. 1990; 65: 57-59.

Haque MA, Golam Nabi PD. Achievements of national TB control program. Bangladesh Med Res Counc Bull. 1999; 25: 71-82.

Haque ME, Yousuf M, Haque AKMS. A study on side effects of thiacetazone. Chest Heart Bull. 1995; 2: 63-65.

Hussain Z, Kar P, Hussain SA. Antituberculosis drug- induced hepatitis; risk factors, prevention and management. Indian J Exp Biol. 2003; 41: 1226-32.

Lee AM, Mennone JZ, Jones RC, Paul WS. Risk factors for hepatotoxicity associated with rifampicin and pyrazina-mide for the treatment of latent tuberculosis infection: Experience from three public health tuberculosis clinics. Int J Tuberc Lung Dis. 2002; 6: 995-1000.

Lee WM. Drug-induced hepatotoxicity. N Engl J Med. 2003; 349: 474-85.

Maddrey WC. Drug-induced hepatotoxicity. J Clin Gastroenterol. 2005; 39: S83-89.

Marti L, Del Olmo JA, Tosca J, Ornia E, Garcia-Torres ML, Serra MA, Rodriguez F, Lluch P, Escudero A, Rodrigo JM. Clinical evaluation of drug-induced hepatitis. Rev Esp Enferm Dig. 2005; 97: 258-65.

Mathus KC, Jain VK, Gupta PP, Jain A. Multiple drug reaction during chemotherapy of tuberculosis. Indian Med Gazette.

\section{9; 8: 274}

Mohan A, Sharma SK. Side effects of anti-tuberculosis drugs. Am J Respir Crit Care Med. 2004; 169: 882-83.

Ohkawa K, Hashiguchi M, Ohno K, Kiuchi C, Takahashi S, Kondo S, Echizen H, Ogata H. Risk factors for antituberculosis chemotherapy-induced hepatotoxicity in Japanese pediatric patients. Clin Pharmacol Ther. 2002; 72: 220-26.

Ormerod LP, Horsfield N. Frequency and type of reactions to anti-tuberculosis drugs: Observation in routine treatment. Tuberc Lung Dis. 1996; 77: 37-42.

Pande JN, Singh SP, Khilnani GC, Khilnani S, Tandon RK. Risk factors for hepatotoxicity from anti-tuberculosis drugs: A case-control study. Thorax 1996; 51: 132-36.

Pereira RM, Tresoldi AT, Hessel G. Isoniazid-induced hepatic failure report of a case. Arq Gastroenteral. 2000; 37: 72-75.

Postlethwaite AE, Bartel AG, Kelley WN. Hyperuricemia due to ethambutol. N Engl J Med. 1972; 286: 761-62.

Resi D, Gangliotti C, Moro ML. Side effects of anti-tuberculosis therapy. Am J Respir Crit Care Med. 2004; 169: 542.

Schaberg T, Rebhan K, Lode H. Risk factors for side-effect of isoniazid, rifampicin and pyrazinamide in patients hospitalized for pulmonary tuberculosis. Eur Respir J. 1996; 9: 2026-30.

Scott JT. Drug-induced gout. Baillieres Clin Rheumatol. 1991; 5: $39-60$.

Shakya R, Rao BS, Shrestha B. Incidence of hepatotoxicity due to anti-tubercular medicines and assessment of risk factors. Ann Pharmacother. 2004; 38: 1074-79.

Smith JM, Zirk MH. Toxic and allergic drug reaction during the treatment of tuberculosis. Tubercle 1961; 42: 287.

Solangi GA, Zuberi BF, Shaikh S, Shaikh WM. Pyrazinamideinduced hyperuricemia in patients taking anti-tuberculosis therapy. J Coll Physicians Surg (Pak). 2004; 14: 136-38.

Thamari JP, Gupta VK. Hypersensitivy of multiple drug in treatment of pulmonary tuberculosis. Indian J Tuberc. 1981; 28: 143 .

Tost JR, Vidal R, Cayla J, Diaz-cabanela D, Jimenez A, Broquetas JM. Study group for severe hepatotoxicity due to anti-tuberculosis Drugs. Int J Tuberc Lung Dis. 2005; 9: 53440.

Tsai RK, Lee YH. Reversibility of ethambutol optic neuropathy. J Ocul Pharmacol Ther. 1997; 13: 473-77.

Van leuven M, De Groot M, Shean K. Pulmonary resection as an adjunct in the treatment of multidrug resistant tuberculosis. Ann Throac Surg. 1997; 63: 1370-72.

Vandeun A, Portalels F. Limitation and requirement for quality control of sputum smear microscopy for AFB. Int J Tuberc Lung Dis. 1998; 2: 756-57.

WHO. Guide line for the management of drug resistant tuberculosis. 1998, pp 5-8.

WHO. Standardized treatment regimens. Treatment of 
tuberculosis. Guide lines for national programs, WHO/ TB. $2^{\text {nd }}$ ed. 1997, pp 23-31.

WHO. TB deaths reach historic levels. Press release WHO; 1996, pp 1-3.

WHO. Treatment of tuberculosis guidelines for national program $3^{\text {rd }}$ ed. Geneva, WHO/CDS/TB/ 2003, p 313.
Wright PW, Wallace RJ Jr, Write NW, Brown BA, Griffith DE. Sensitivity of flurochrome microscopy for detection of mycobacteria. J Clin Microbial. 1998; 4: 1046-49.

Zierski M, Bek E. Side-effect of drug regimens used in short course chemotherapy for pulmonary tuberculosis a controlled clinical study. Tubercle 1980; 61: 41-49.

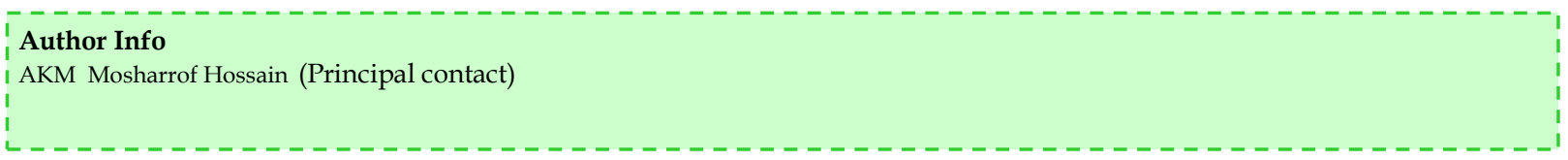

